\title{
Usefulness of Posterolateral Transforaminal Approach in Lumbar Radicular Pain
}

\author{
Ji Woong Park, M.D., Hee-Seung Nam, M.D. ${ }^{1}$, Yongbum Park, M.D. ${ }^{2}$
}

\begin{abstract}
Department of Rehabilitation Medicine, Soonchunhyang University College of Medicine, Seoul 140-887, ${ }^{1} \mathrm{Hallym}$ University College of Medicine, Seoul 134-814, ${ }^{2}$ Sanggye Paik Hospital, Inje University College of Medicine, Seoul 139-707, Korea
\end{abstract}

Objective To compare the short-term effects and advantages of transforaminal epidural steroid injection (TFESI) performed using the conventional (CL) and posterolateral (PL) approaches.

Method Fifty patients with lumbar radicular pain from lumbar spinal stenosis and herniated lumbar disc were enrolled. Subjects were randomly assigned to one of two groups (CL or PL group). All procedures were performed using a C-arm (KMC 950, KOMED, Kwangju, Kyunggi, Korea). We compared the frequency of complications during the procedure and the effects of the pain block between the two groups at 2, 4, and 12 weeks after the procedure.

Results There were no significant differences in the demographic data, initial VNS (Visual numeric scale), or ODI (Oswestry disability index) between the CL group $(n=26)$ and the PL group $(n=24)$. There was no statistically significant difference in the outcome measures (VNS and ODI) between the groups at 2, 4, or 12 weeks. Symptoms of nerve root irritation occurred in 1 case of the CL group and in 7 cases of the PL group $(\mathrm{p}<0.05)$. Pricking of spinal nerve during the procedure and transient weakness after the procedure occurred in 6 cases and 3 cases, respectively in the CL group, but did not occur in the PL group.

Conclusion Our findings suggest that the posterolateral approach represents an alternative TFESI method in cases with difficult needle tip positioning in the anterior epidural space, and could lower the risk of target nerve root irritation and nerve penetration.

Key Words Transforaminal, Conventional, Posterolateral, Injection

\section{INTRODUCTION}

Steroid injections have been used for the treatment of lumbar radicular pain since 1952. ${ }^{1}$ There are three technically different injection methods to access the

Received July 21, 2010; Accepted November 28, 2010

Corresponding author: Yongbum Park

Department of Rehabilitation Medicine, Sanggye Paik Hospital, Inje University College of Medicine, 761-1, Sanggye 6,7 dong, Nowon-gu, Seoul 139-707, Korea

Tel: +82-2-950-1390, Fax: +82-2-935-3076, E-mail: swc328@naver.com Copyright $\odot 2011$ by Korean Academy of Rehabilitation Medicine epidural space: the interlaminar approach, the caudal approach, and the transforaminal approach. In theory, the transforaminal approach allows the injection needle to reach the closest possible position to either the distal nerve root or the anterior portion of the epidural space. Thus, a low-volume drug can be administered at a high concentration to a specific region, and it is likely to provide better treatment effects for a longer period of time, compared to the other two approaches, which were mainly used in the past. ${ }^{2-4}$ In the conventional transforaminal approach, an injection needle is 
advanced into the safe triangle under the inferior aspect of the pedicle, as described by Bogduk ${ }^{5}$ and positioned superolaterally to the exiting spinal nerve. In this position, a drug can be injected either into the anterior epidural space or the site of inflammation between the posterior aspect of the herniated disc and the anterior aspect of the dural nerve-root sleeve. Since the needle passes through the superolateral portion of the neural foramen, there is less risk of damaging the dura mater. ${ }^{6,7}$ However, Lee et al. ${ }^{8}$ reported that when a conventional transforaminal approach is used, the injection needle should be placed in the anterior portion of the neural foramen across the nerve root. Actually, it is often difficult to do so, especially in cases of severe foraminal stenosis. Furthermore, as the needle should be directed to the anterior epidural space across the neural foramen, it may cause damage to the nerve roots. Many arterial blood vessels are distributed throughout the space, thus there is a risk of severe complications (e.g., paraplegia by spinal cord infarction). ${ }^{9-11}$ Many other injection approaches have been introduced as alternatives to the convention al transforaminal approach. As one of such alternative option, the posterolateral transforaminal approach proposed by Lee et al. ${ }^{8}$ carries less risk of drug infusion into the anterior radicular artery or of damage to nerve roots, because the needle is placed in the posterolateral portion of the neural foramen. However, the study by Lee et al. ${ }^{8}$ had its limitations because it was retrospectively conducted and investigated the diagnostic and treatment effects over a period of only 2 weeks. Therefore, the present study used a prospective randomized method in patients diagnosed with lumbar radicular pain. The purpose was to compare the conventional transforaminal approach and the posterolateral transforaminal approach in terms of their treatment effects, injection procedures, and possible post-treatment complications over a period of 3 months.

\section{MATERIALS AND METHODS}

The subjects were patient volunteers who decided to participate in this study being informed about its purpose, the procedures involved, and the potential risks and complications.

\section{Subjects}

The subjects of this study were selected from those patients who visited our hospital's Department of Physical Medicine and Rehabilitation with a complaint of lumbar radiculopathy between June 1, 2009 and May 31, 2010, and received transforaminal injections to treat their condition. The patients were considered eligible when meeting the following inclusion criteria:

(1) Suspected cases of lumbar nerve-root compression, as assessed by clinical examination, medical interview or electromyography; or (2) Cases of herniated disc or spinal stenosis detected by either lumbar CT or MRI of the nerve root.

The exclusion criteria were as follows:

(1) Patients with a systematic inflammatory disease

(2) Patients taking an anticoagulant

(3) Cases of diabetes that were likely to develop side effects from steroid use

(4) Patients who had previously experienced a side effect from the use of lidocaine or contrast medium

(5) Patients with a known or suspected infectious disease

(6) Patients who found it difficult to visit the hospital because of general bad health

(7) Patients with a skin disorder unsuitable for administration of injection at the injection site

(8) Patients with a mental health problem who were unable to complete a questionnaire

(9) Patients who had received injection treatment in the same region in the past 3 months

(10) Patients who continued to take an anti-inflammatory analgesic other than acetaminophen, or a drug that was likely to influence the assessment of treatment effects, or received such a treatment (e.g., physical therapy) during the clinical trial

(11) Cases of cauda equine syndrome or a similar disorder.

\section{Randomization}

The investigator randomly assigned the subjects to one of two groups after obtaining information on their basic characteristics. The subjects received injections by one of the following two approaches:

(1) Conventional transforaminal approach: 1 cc +2 cc (1.5 $\mathrm{ml}$ of $0.5 \%$ lidocaine $+20 \mathrm{mg}$ of triamcinolone) of nonionic contrast medium (Omnipaque 300; GE Healthcare, 
Carrigtohill, Co. Cork, Ireland)

(2) Posterolateral transforaminal approach: $1 \mathrm{cc}+2 \mathrm{cc}(1.5$ $\mathrm{ml}$ of $0.5 \%$ lidocaine $+20 \mathrm{mg}$ of triamcinolone) of nonionic contrast medium (Omnipaque 300; GE Healthcare, Carrigtohill, Co. Cork, Ireland)

Two weeks after the first injection treatment, the patients who showed no effect were excluded. However, the patients showing only partial effect, e.g. $<50 \%$ measured on the visual numeric scale (VNS), or even $<30 \%$ measured on the Oswestry disability index, were given the second injection two weeks later. The subjects were randomized into two groups by an independent investigator who did not have access to the patients' information.

\section{Injection approaches}

Conventional transforaminal injections: Each patient was placed in the prone position with a pillow under the abdomen to reduce the lumbar lordosis, and a Scotty dog shadow was used to identify the desired lumbar region. The $\mathrm{C}$-arm was adjusted to align with the inferior endplate of the spine and rotated by 15-30 degrees to an oblique position, so that the Scotty dog shadow became visible. The target region was disinfected, and a 22-gauge, 3.5-inch spinal needle (Spinocan ${ }^{\circledR}$, BRAUN, Melsungen, Germany) was used. The needle was advanced into the inferior aspect of the pedicle (safe triangle) towards the superior aspect of the neural foramen, in order to avoid the nerve roots emerging from the spinal cord. When the needle tip reached the inferolateral border, the $\mathrm{C}$-arm was rotated to obtain lateral images and the needle was slowly advanced towards the anterior aspect of the neural foramen. Once the needle was placed in the final position, a fine aspiration needle was inserted to determine if blood was detected. One cc of nonionic contrast medium (Omnipaque 300; GE Healthcare, Carrigtohill, Co. Cork, Ireland) was administered under real-time fluoroscopic imaging to determine if the injection reached the anterior epidural space. Every procedure was performed by the same surgeon among the authors. After postero-anterior and lateral radiographs were taken during contrast administration, a $2 \mathrm{cc}$ drug solution $(1.5 \mathrm{ml}$ of $0.5 \%$ lidocaine $+20 \mathrm{mg}$ of triamcinolone) was injected.

Posterolateral transforaminal injections : The posterolateral transforaminal approach used in this study was similar to the approach proposed by Lee et al. ${ }^{8}$ According to them, when using the conventional transforaminal approach, because the drug tends to flow into the distal portion along spinal nerves, the injection needle on an oblique view should be placed in the region where a nerve root passes slightly more inferomedially to the pedicle. However, the needle should be positioned in the posterior portion on a lateral view and in the
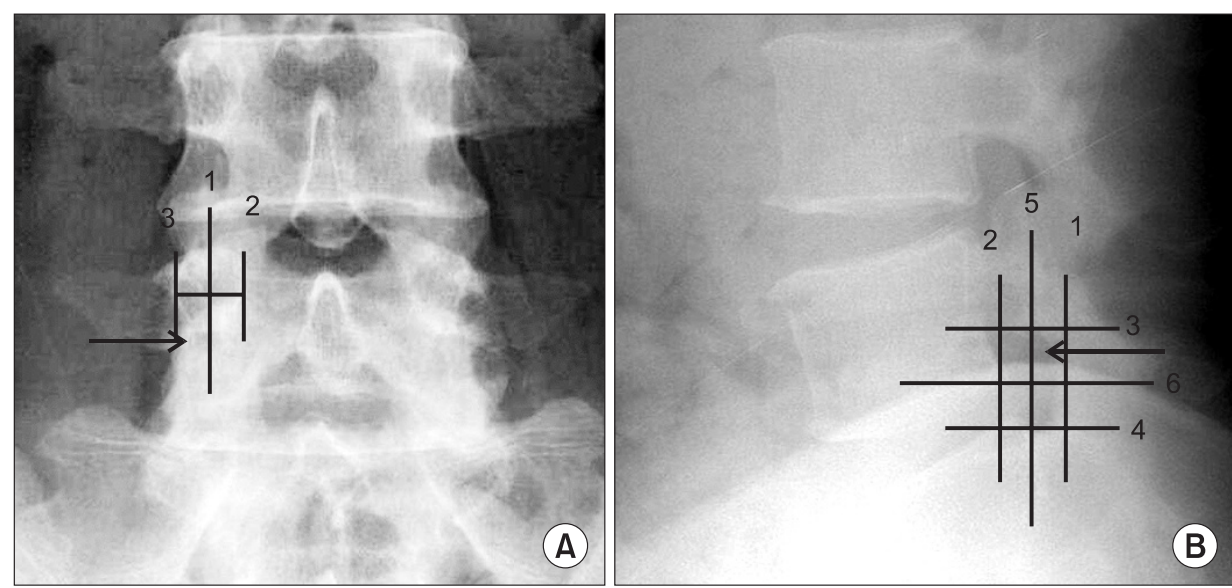

Fig. 1. (A) Anterior-posterior view of the lumbar spine, with superimposed line (1) bisecting the pedicle. This line was drawn halfway between the farthest medial (2) and farthest lateral (3) points on the pedicle. (B) Lateral view of the lumbar spine, with the quadrant system superimposed. First, a line was drawn tangent to the curve of the spine at the level of interest along the posterior vertebral line. (1) A second line (2) was drawn parallel to a this at the posterior margin of the foramen. Next, 2 lines perpendicular to lines 1 and 2 were drawn at the superior and inferior margins of the foramen ( 3 and 4, respectively). Finally, line (5) was drawn bisecting 1 and 2, and likewise line (6) bisecting 3 and 4. This divided the foramen into 4 quadrants. Arrow: needle position. 

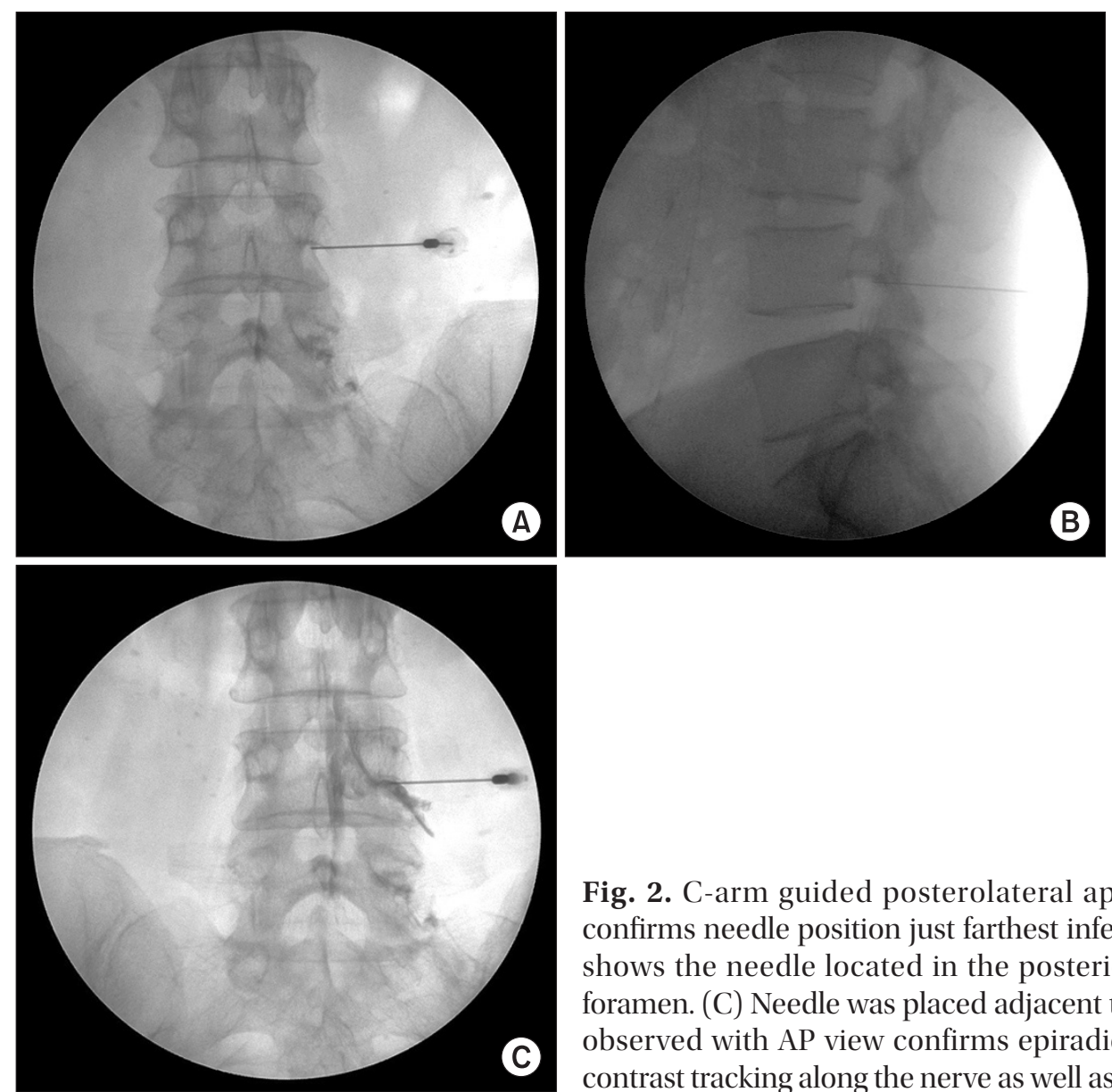

Fig. 2. C-arm guided posterolateral approach. (A) AP fluoroscopic image confirms needle position just farthest inferior-lateral of pedicle. (B) Lateral view shows the needle located in the posterior and superior of the intervertebral foramen. (C) Needle was placed adjacent to the $\mathrm{L} 4$ nerve root. Contrast injection observed with AP view confirms epiradicular location of the needle's tip with contrast tracking along the nerve as well as entering the epidural space locally.

lateral portion under the pedicle on a postero-anterior radiograph. This approach allows the doctor to guide the injection needle into a target site while seeing oblique and lateral views at the same time, using the bi-planar $\mathrm{C}$-arm. Therefore, on the oblique view, the needle can be safely advanced into the inferomedial aspect of the respective pedicle, through which a nerve root passes. However, we lacked the required equipment and thus had to continue to observe the lateral view while injecting. Because this was likely to cause prolonged exposure to radiation and damage the nerve root, we placed the needle at the 6 o'clock position of the pedicle on an oblique view, similar to the conventional transforaminal approach. To prevent both damage to the dural sleeve and drug infusion into the subarachnoid space, and to reduce vascular injuries and discomfort from injection, we placed the needle on a site off the lateral region, with the pedicle bisected on postero-anterior radiographs and placed in the postero-superior portion on lateral view. The neural foramen was divided into four equal parts on the lateral radiographs, using the method suggested by Crall et al. ${ }^{12}$ to distinguish the neural foramen (Fig. 1). Once the needle tip was in its final position, the investigator administered $1 \mathrm{cc}$ of nonionic contrast (Omnipaque 300; GE Healthcare, Carrigtohill, Co. Cork, Ireland) under real-time fluoroscopic imaging to observe the spreading range and patterns of the contrast medium. We then injected the prepared $2 \mathrm{cc}$ drug solution $(1.5 \mathrm{ml}$ of $0.5 \%$ lidocaine $+20 \mathrm{mg}$ of triamcinolone) (Fig. 2).

\section{Comparison of injection results between the two approaches}

The complications that occurred during and after injections were recorded for comparison. To compare the effects of the injection treatment, we determined the pain intensity using the visual numeric rating scale (VNS), at baseline and at weeks 2, 4, and 12 of treatment. A pain measuring tool within a $100 \mathrm{~mm}$ visual line was shown to patients. Pain was rated on an 11-point scale, ranging from no pain $=0$, to worst severe pain $=10$. The patients 
were asked to indicate their pain level on the scale. For patients who found it difficult to rate their pain, the investigator moved a pointer slowly along the scale, from the 0 point to the opposite direction. The patients were asked to nod their heads when the pointer reached an appropriate position. The degrees of physical disability were measured using the Oswestry Disability Index (ODI), which is a measure designed to assess physical disability. In this study, physical disability was presented as percentage levels ranging from no disability $=0 \%$ to most severe disability $=100 \%$. The subjects were asked to complete a questionnaire, and based on the questionnaire results, the disability levels of the patients were assessed at baseline and at weeks 2, 4, and 12 of treatment.

\section{Statistics}

The chi-square test and the independent t-test were used to compare the two groups of patients in terms of age, sex, body mass index (BMI), disease duration, injection frequency, and radiologic results. The independent t-test was conducted to compare the ODI scores of the VNS between the two groups at each treatment time point. A repeated measures analysis of variance (ANOVA) was used to assess injection effects over time, and Bonferroni's correction was applied for post hoc comparisons. The Fisher's exact test was performed for comparisons of possible complications and injection-related discomfort in the two groups. Data were statistically analyzed with a significance level of $p \leq 0.05$, using the SAS Enterprise Guide, version 4.1 (4.1.0.471) software.

\section{RESULTS}

\section{General characteristics of the subjects}

This study included a total of 50 subjects. They were grouped to undergo one of the two injection approaches as follows: 26 subjects were assigned to the "conventional transforaminal approach" group (mean age $=52.50 \pm 9.94$ years) and 24 were assigned to the "posterolateral transforaminal approach" group (mean age $=53.50 \pm 9.09$ years). There was no statistically significant difference between the two groups in the following measurements: male-female ratio, body mass index, painful duration, injection frequency, lesion site, and radiologic results (Table 1).

\section{Patient flow}

A total of 66 patients were enrolled as subjects in this study. Seven were dropped from the study according to our exclusion criteria. Two subjects in the posterolateral group were excluded from the study because they showed no treatment effect at week 2 . In the middle of the study, one subject from the posterolateral group and two from the conventional group were dropped because they transferred to a different medical department for surgical

Table 1. Characteristics of the Patients

\begin{tabular}{lccc}
\hline & Conventional approach $(\mathbf{n}=\mathbf{2 6})$ & Posterolateral approach $(\mathbf{n}=\mathbf{2 4})$ & p-value \\
\hline Age (year) & $52.50 \pm 9.94$ & $53.50 \pm 9.09$ & 0.712 \\
Male & $11(42.3 \%)$ & $11(45.8 \%)$ & 0.802 \\
Female & $15(57.3 \%)$ & $13(54.2 \%)$ & 0.856 \\
BMI $\left(\mathrm{kg} / \mathrm{m}^{2}\right)$ & $23.26 \pm 1.46$ & $23.34 \pm 1.49$ & 0.665 \\
Duration (Month) & $7.77 \pm 2.79$ & $7.42 \pm 2.92$ & 0.926 \\
No. of injection & $1.35 \pm 0.485$ & $1.33 \pm 0.482$ & 0.924 \\
Spinal stenosis & $17(65.4 \%)$ & $16(66.7 \%)$ & \\
HLD & $9(34.6 \%)$ & $8(33.3 \%)$ & \\
Injection level & $1(3.8 \%)$ & $0(0 \%)$ & \\
L2-3 & $2(7.7 \%)$ & $1(4.2 \%)$ & 0.651 \\
\hline L3-4 & $9(34.6 \%)$ & $7(29.2 \%)$ & $16(66.7 \%)$ \\
\hline L4-5 & $14(53.8 \%)$ & & \\
\hline L5-S1 & & & \\
\hline
\end{tabular}

Values are mean \pm standard deviation

BMI: Body mass index, HLD: Herniated lumbar disc 


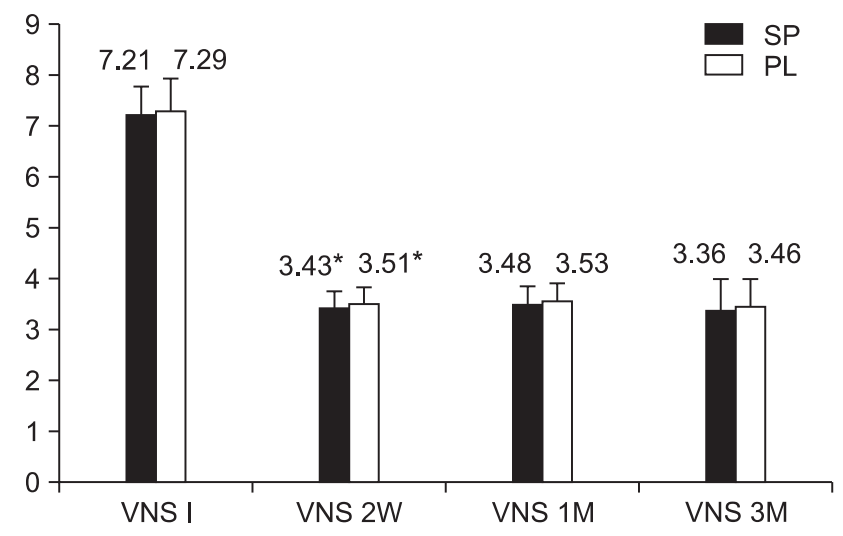

Fig. 3. Comparative response from VNS preinjection, 2 weeks, 1 month, 3 months for conventional approach and posterolateral approach. ${ }^{*}$ Statistically significant at $\mathrm{p}<0.05$ within groups.

treatment. One patient in the conventional group was also dropped for taking a drug other than acetaminophen during the study. In addition, three other patients were excluded halfway through the study, as it became difficult for them to regularly visit the hospital.

\section{Treatment effects}

At weeks 2, 4, and 12 of treatment, as well as at baseline, there was no statistically significant difference between the two investigated groups with regard to the ODI and the VNS (Fig. 3, 4). For the treatment effectiveness over time after the injection procedure, both groups showed a significant decrease in the VNS score compared to baseline. The effect was maintained until weeks 4 and 12 of treatment (Fig. 3). The ODI was also significantly improved in both groups, compared to baseline, and such improvement continued until weeks 2,4 , and 12 of treatment (Fig. 4).

Injection procedures and post-treatment complications During drug injection, we found nerve root irritation in seven cases from the conventional group and one case in the posterolateral group. During needle insertion, we found nerve-root pricking in six cases, all from the conventional group. As a result, there were significant differences between the two groups in the number of such cases. Although three cases of intravascular infusion were observed only in the conventional group, there was no statistically significant difference between the two groups. Muscle weakness caused after treatment

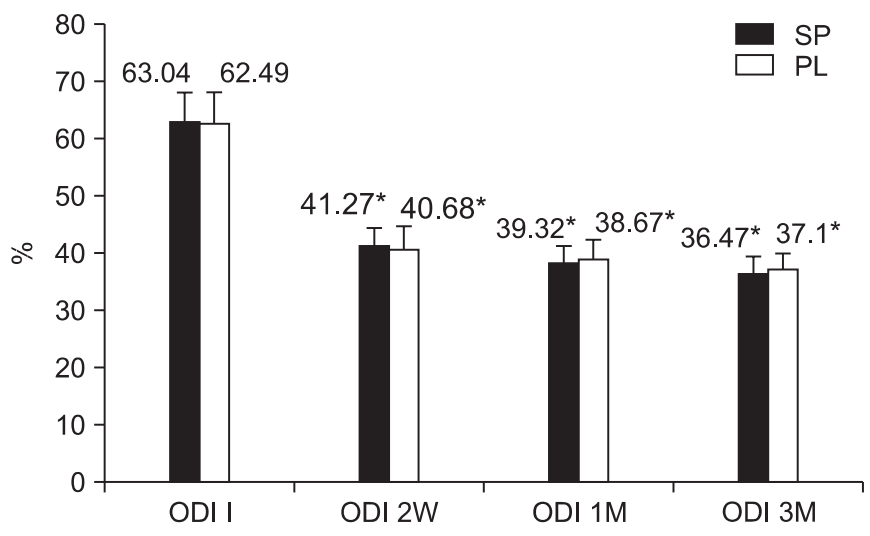

Fig. 4. Comparative response from ODI preinjection, 2 weeks, 1 month, 3 months for conventional approach and posterolateral approach. *Statistically significant at $\mathrm{p}<0.05$ within groups.

Table 2. Forte and Foible during Procedure

\begin{tabular}{lccc}
\hline & $\begin{array}{c}\text { CL } \\
(\mathbf{n = 2 6 )})\end{array}$ & $\begin{array}{c}\text { PL } \\
(\mathbf{n = 2 4 )}\end{array}$ & p-value* \\
\hline Root irritation (\%) & $7(26.9 \%)$ & $1(4.2 \%)$ & 0.050 \\
Spinal nerve pricking (\%) & $6(23.1 \%)$ & $0(0 \%)$ & 0.023 \\
Intra-vascular injection (\%) & $3(11.5 \%)$ & $0(0 \%)$ & 0.236 \\
Transient weakness (\%) & $3(11.5 \%)$ & $0(0 \%)$ & 0.236 \\
Dural puncture & $0(0 \%)$ & $0(0 \%)$ & \\
\hline
\end{tabular}

CL: Conventional approach, PL: Posteriolateral approach ${ }^{*}$ Compared by Fisher's exact test

was found in three cases, all in the conventional group; however, for this measurement, there was no statistically significant difference between the two groups. Dural puncture and other similar complications did not occur in either of the studied groups during the treatment procedures (Table 2). After the transfer of subjects to the recovery room, the progress of all complications was observed. No subject showed any complication when discharged from the hospital.

\section{DISCUSSION}

One important possible cause of nerve root irritation is the mechanical compression exerted by degenerative changes in the intervertebral disc, the posterior longitudinal ligament or the intervertebral joint. Another cause is chemical irritation produced by phospholipase A2 or by the substance P secreted from the herniated 
disc. As a result, they contribute to the pain generation by triggering venous congestion and/or neural edema around the nerve root. ${ }^{13-15}$

Many different causes of pain have been known or reported. When injected in the human body, steroids ease pain by inhibiting prostaglandin synthesis, blocking the conduction of nociceptive C-fibers, and controlling the edema around nerve roots. ${ }^{16-19}$ For this reason, steroid injections are used in patients with herniated disc or lumbar spinal stenosis.

The conventional transforaminal approach allows a high dose of steroid or anesthetic preparation to be effectively injected into the anterior epidural space through the safe triangle described by Bogduk ${ }^{5}$ and around the nerve root and the proximal portion of the intervertebral disc. Since the neural structures are typically located at the medial aspect of neural foramen, neurological complications can occur as the injection needle is medially advanced. ${ }^{20}$ Bogduk et al. ${ }^{21}$ proposed that if injections were given through the safe triangle, they could reduce the occurrence of neurological complications such as severe loss of disc height or spondylosis, which is the deformation of the anatomic structures that constitute the safe triangle. Injections given through the safe triangle can trigger complications, such as dural tears and subarachnoid drug infusion. ${ }^{22}$ In the anatomical relations, the dorsal root ganglia are reported to be located immediately below the pedicle in $90 \%$ of the cases, in the medial portion of the pedicle in $2 \%$, and below the lateral aspect of the pedicle in $8 \%{ }^{23}$ Therefore, even in a normal state where there is no deformation of the anatomic structures constituting the intervertebral foramen, the injections made through the safe triangle can't ensure safety. In this study, according to the injection approach proposed by Crall et al., ${ }^{12}$ the pedicle was bisected on a postero-anterior view and the needle was placed for drug injection in its lateral segment.

Complications such as dural tears and subdural drug infusion were not found in any of the two groups. This may have happened because the sample size was too small, similar to findings of a previous study ${ }^{24}$ that reported an incidence of $0.82 \%$ subdural injections during the use of the interlaminar injection approach; thus, further larger-scale studies are required.

Lee et al. ${ }^{8}$ suggested that when the conventional transforaminal approach is performed in cases of severe foraminal stenosis, there is a risk of pricking the spinal nerve roots during the injection. This happens because the injection needle is positioned in the anterior portion of the neural foramen across the nerve roots. In contrast, the posterolateral transforaminal approach carries less risk of pricking because the needle is positioned in the superior-posterior aspect on a lateral radiograph. Similarly, in our study, the conventional transforaminal approach produced needle pricking in six subjects with severe foraminal stenosis and severe loss of disc height, but there was no such event when the posterolateral transforaminal approach was used $(\mathrm{p}<0.05)$.

The incidence of intravascular drug infusion in the transforaminal approach is reported to be $9-26 \%$, depending on the location of the injection needle. ${ }^{25-28}$ Derby et al. ${ }^{29}$ reported an incidence of $0.56 \%$ for intra-arterial drug infusion, which can lead to severe disability. The Adamkiewicz artery supplies blood to the inferior $2 / 3$ of the spinal cord, branches out from the aorta, and enters the spinal canal through one of the T7-L2 intervertebral foramina. When entering into the foramen, it passes in close proximity to the dorsal root ganglia. In $-80 \%$ of cases, the Adamkiewicz artery is located on the left side of T9-L1, the superior or middle portion of the neural foramen, and the anterior superolateral aspect of the dorsal root ganglion. ${ }^{30}$ Therefore, as the conventional transforaminal approach has the needle placed on the anterior superolateral aspect of the neural foramen, the use of this injection approach can cause direct damage to the Adamkiewicz artery or can trigger complications, such as spinal infarction by intravascular injection of particulate steroids. In contrast, the posterolateral transforaminal approach has the needle placed on the postero-superior aspect of the neural foramen and can reduce the risk of such complications. However, when a drug is injected into blood vessels that constitute a venous plexus in the posterolateral aspect of the epidural space, it is difficult to achieve the desired treatment effect because of systemic administration. Accordingly, the intravascular spread of contrast medium should be monitored even when using the posterolateral injection approach. In this study, although there was no statistically significant difference between the two groups, the accidental intravascular infusion of contrast was found in three cases in the conventional approach group, but no cases were found in the posterolateral 
approach group. Even though the contrast medium was injected not into arterial, but into venous vessels in both groups, the above finding suggests that proper care should be taken with injection.

Among minor complications that occur after a foraminal injection, the radicular pain may become severe during or after the injection procedure. Radicular pain has been reported with an average incidence of $4 \% .{ }^{31,32}$ Such pain occurs as a large dose of drug is injected into the spinal canal, previously narrowed due to herniated disc, spinal canal stenosis, or epidural fibrosis. ${ }^{33}$ Pfirrmann et al. ${ }^{34}$ proposed the lateral region of the safe triangle as the ideal needle location, producing less pain during treatment. Lee et $\mathrm{al}^{8}{ }^{8}$ reported that when using the posterolateral transforaminal approach, patients were less likely to complain of discomfort compared with the conventional approach. Pain can be minimized when a drug injection is given slowly, at a rate of $0.25 \mathrm{cc}$ per second. ${ }^{35}$ However, nerve root irritation is not always significant. Usually, if the medication has not been injected into the nerve root sheath, it means that the needle has been inserted into the correct site. There is a disadvantage, i.e., the patients might feel radicular pain during the injection procedure. However, they are expected to get the best possible effect from the treatment via posterolateral transforaminal injections. In this study, eight subjects complained of radicular pain during the drug injection: seven cases in the conventional group and one case in the posterolateral group $(\mathrm{p}<0.05)$.

A fibrotic band divides the posterior aspect of the epidural space into the right and left sides, and it extends from the dorsal side of the dura to the ligamentum flavum. We named this band the "plica mediana dorsalis." ${ }^{36}$ There is also connective tissue extending from the fibrotic structure into both sides, and the posterior aspect of the epidural space is redivided into two parts. When an interlaminar approach is used, it sometimes prevents a posteriorly injected drug from going into the anterior epidural space. ${ }^{37}$ Our study found no symptom improvement in two subjects who received a posterolateral transforaminal injection. Two weeks later, when a conventional transforaminal injection was performed in the same subjects, both of them showed pain relief of $>50 \%$. Manchikanti et al. ${ }^{5}$ reported in their study of 100 patients that the spread of contrast into the posterior epidural space was found in $9 \%$ of 256 conventional transforaminal injections. The contrast medium injected into the epidural space diffused through the region of lowest resistance. Based on these findings, they suggested that when there is a herniated disc or severe spinal stenosis, medications must not be injected into the anterior epidural space, because of the strong resistance of the space resulting from such a condition. As a result, an injected drug will stay in the posterior epidural space due to the strong resistance of the anterior epidural space and/or other anatomic structures, such as the above mentioned plica mediana dorsalis. This is probably the reason why we found no treatment effect from the first injection in those two subjects presented above.

In the posterolateral transforaminal approach, as medications are injected into the posterolateral epidural space, they are not likely to enter the anterior epidural space, which is the lesion. Conceivably, this can reduce the treatment effects, but the present study found no statistically significant difference. When observing the progress of patients for two weeks after an injection, Lee et al. ${ }^{8}$ also reported that there was no difference between the two injection approaches with regard to the effectiveness of the diagnostic treatment. Crall et al. ${ }^{12}$ conducted a retrospective study in which they divided the intervertebral foramen into four parts and assessed the treatment effects immediately after giving an injection to each part. They reported no statistically significant difference between the conventional and the posterolateral approaches.

Similarly, we did not find any significant difference in the treatment effectiveness, but the study results indicate that when the posterolateral transforaminal approach was performed with a 2-cc injection dose and drug concentration, it was likely to provide sufficient medication effects by overcoming the distance from the posterolateral epidural space to the anterior epidural space of the lesion site. However, when using the posterolateral approach, a drug cannot be injected at a high concentration into the anterior epidural space, compared with the conventional approach. Therefore, the progress assessment over 3 months may not be enough for comparison of the treatment effects. Future long-term studies of 6 or more months are required to examine the interactions between treatment effects and either drug dosage or injection sites. 
This study was limited in that the number of subjects (sample size) was too small; and furthermore, it did not consider the interactions between treatment effects and factors that can affect the pain index (i.e., results of physical exam, patient mood, general health measures, and smoking status). Future studies need to be conducted as long-term attempts to overcome such limitations.

\section{CONCLUSION}

The posterolateral transforaminal approach is an alternative option to the conventional transforaminal method, when it is difficult to place the needle in the anterior epidural space.

Comparatively, it can reduce both nerve root irritation and patient discomfort, and it carries less risk of pricking the spinal nerve roots.

\section{REFERENCES}

1. Slipman CW, Chow DW. Therapeutic spinal corticosteroid injections for the management of radiculopathies. Phys Med Rehabil Clin N Am 2002; 13: 697-711

2. Manchikanti L, Staats PS, Singh V, Schultz DM, Vilims BD, Jasper JF, Kloth DS, Trescot AM, Hansen HC, Falasca TD, et al. Evidence-based practice guidelines for interventional techniques in the management of chronic spinal pain. Pain Physician 2003; 6: 3-81

3. Abdi S, Datta S, Trescot AM, Schultz DM, Adlaka R, Atluri SL, Smith HS, Manchi kanti L. Epidural steroids in the management of chronic spinal pain: a systematic review. Pain Physician 2007; 10: 185-212

4. Manchikanti L. Transforaminal lumbar epidural steroid injections. Pain Physician 2000; 3: 374-398

5. Bogduk N. Clinical anatomy of the lumbar spine and sacrum. 4th ed. New York, churchill Livingstone: 2005, 123-124

6. Eriksen $\mathrm{W}$. The prevalence of musculoskeletal pain in Norwegian nurses' aides. Int Arch Occup Environ Health 2003; 76: 625-630

7. Botwin KP, Gruber RD, Bouchlas CG, Torres-Ramos FM, Sanelli JT, Freeman ED, Slaten WK, Rao S. Fluoroscopically guided lumbar transforaminal epidural steroid injections in degenerative lumbar stenosis: an outcome study. Am J Phys Med Rehabil
2002; 81: 898-905

8. Lee IS, Kim SH, Lee JW, Hong SH, Choi JY, Kang HS, Song JW, Kwon AK. Comparison of the temporary diagnostic relief of transforaminal epidural steroid injection approaches: conventional versus posterolateral technique. AJNR Am J Neuroradiol 2007; 28: 204-208

9. Houten JK, Errico TJ. Paraplegia after lumbosacral nerve root block: report of three cases. Spine J 2002; 2: 70-75

10. Somayaji HS, Saifuddin A, Casey AT, Briggs TW. Spinal cord infarction following therapeutic computed tomography-guided left L2 nerve root injection. Spine 2005; 30: E106-108

11. Stretanski MF, Chopko B. Unintentional vascular uptake in fluoroscopically guided, contrast-confirmed spinal injections: a 1-yr clinical experience and discussion of findings. Am J Phys Med Rehabil 2005; 84: 30-35

12. Crall TS, Gilula LA, Kim YJ, Cho Y, Pilgram T, Riew KD. The diagnostic effect of various needle tip positions in selective lumbar nerve blocks: an analysis of 1202 injections. Spine 2006; 31: 920-922

13. Nygaard OP, Mellgren SI, Osterud B. The inflammatory properties of contained and noncontained lumbar disc herniation. Spine 1997; 22: 2484-2488

14. Franson RC, Saal JS, Saal JA. Human disc phospholipase A2 is inflammatory. Spine 1992; 17 Suppl 6: S129-132

15. Olmarker K, Blomquist J, Stromberg J, Nannmark U, Thomsen P, Rydevik B. Inflammatogenic properties of nucleus pulposus. Spine 1995; 20: 665-669

16. Rydevik B, Brown MD, Lundborg G. Pathoanatomy and pathophysiology of nerve root compression. Spine 1984; 9: 7-15

17. Kantrowitz F, Robinson DR, McGuire MB, Levine L. Corticosteroids inhibit prostaglandin production by rheumatoid synovia. Nature 1975; 258: 737-739

18. Fukusaki M, Kobayashi I, Hara T, Sumikawa K. Symptoms of spinal stenosis do not improve after epidural steroid injection. Clin J Pain 1998; 14: 148151

19. Johansson A, Hao J, Sjolund B. Local corticosteroid application blocks transmission in normal nociceptor C-fibres. Acta Anaesthesiol Scand 1990; 34: 335-338 20. Aprill CN, Melfi RS. Paraplegia after lumbosacral 
nerve block: report of three cases. Spine J 2004; 4: 368369

21. Bogduk N, Wilson AS, Tynan W. The human lumbar dorsal rami. J Anat 1982; 134: 383-397

22. Goodman BS, Bayazitoglu M, Mallempati S, Noble BR, Geffen JF. Dural puncture and subdural injection: a complication of lumbar transforaminal epidural injections. Pain Physician 2007; 10: 697-705

23. Hamanishi C, Tanaka S. Dorsal root ganglia in the lumbosacral region observed from the axial views of MRI. Spine 1993; 18: 1753-1756

24. Lehmann LJ, Pallares VS. Subdural injection of a local anesthetic with steroids: complication of epidural anesthesia. South Med J 1995; 88: 467-469

25. Sullivan WJ, Willick SE, Chira-Adisai W, Zuhosky J, Tyburski M, Dreyfuss P, Prather H, Press JM. Incidence of intravascular uptake in lumbar spinal injection procedures. Spine 2000; 25: 481-486

26. Furman MB, O'Brien EM, Zgleszewski TM. Incidence of intravascular penetration in transforaminal lumbosacral epidural steroid injections. Spine 2000; 25: 2628-2632

27. Furman MB, Giovanniello MT, O'Brien EM. Incidence of intravascular penetration in transforaminal cervical epidural steroid injections. Spine 2003; 28: 21-25

28. Smuck M, Fuller BJ, Yoder B, Huerta J. Incidence of simultaneous epidural and vascular injection during lumbosacral transforaminal epidural injections. Spine J 2007; 7: 79-82

29. Derby R, Lee SH, Kim BJ, Chen Y, Seo KS. Complications following cervical epidural steroid injections by expert interventionalists in 2003. Pain Physician 2004;
7: 445-449

30. Alleyne CH Jr, Cawley CM, Shengelaia GG, Barrow DL. Microsurgical anatomy of the artery of Adamkiewicz and its segmental artery. J Neurosurg 1998; 89: 791795

31. Burn JM, Langdon L. Lumbar epidural injection for the treatment of chronic sciatica. Rheum Phys Med 1970; 10: 368-374

32. Snoek W, Weber H, Jorgensen B. Double blind evaluation of extradural methyl prednisolone for herniated lumbar discs. Acta Orthop Scand 1977; 48: 635-641

33. Botwin KP, Gruber RD, Bouchlas CG, Torres-Ramos FM, Freeman TL, Slaten WK. Complications of fluoroscopically guided transforaminal lumbar epidural injections. Arch Phys Med Rehabil 2000; 81: 1045-1050

34. Pfirrmann CW, Oberholzer PA, Zanetti M, Boos N, Trudell DJ, Resnick D, Hodler J. Selective nerve root blocks for the treatment of sciatica: evaluation of injection site and effectiveness: a study with patients and cadavers. Radiology 2001; 221: 704-711

35. Bogduk N. Epidural steroids. Spine 1995; 20: 845-848

36. Huson A, Luyendijk W, Tielbeek A, Van Zundert A. CT-epidurography and the anatomy of the human lumbar epidural space. Anesthesiology 1988; 69: 797798

37. Thomas E, Cyteval C, Abiad L, Picot MC, Taourel P, Blotman F. Efficacy of transforaminal versus interspinous corticosteroid injection in discal radiculalgiaa prospective, randomized, double-blind study. Clin Rheumatol 2003; 22: 299-304 